\title{
AN ARITHMETICAL EXCURSION VIA STONEHAM NUMBERS
}

\author{
MICHAEL COONS \\ To Professor Peter Borwein on his 60th birthday
}

\begin{abstract}
Let $p$ be a prime and $b$ a primitive root of $p^{2}$. In this paper, we give an explicit formula for the number of times a value in $\{0,1, \ldots, b-1\}$ occurs in the periodic part of the base- $b$ expansion of $1 / p^{m}$. As a consequence of this result, we prove two recent conjectures of Francisco Aragón Artacho, David Bailey, Jonathan Borwein, and Peter Borwein concerning the base- $b$ expansion of Stoneham numbers.
\end{abstract}

\section{INTRODUCTION}

Let $b \geqslant 2$ be an integer. A real number $\alpha \in(0,1)$ is called $b$-normal if in the base $b$ expansion of $\alpha$ the asymptotic frequency of the occurrence of any word $w \in\{0,1, \ldots, b-1\}^{*}$ of length $n$ is $1 / b^{n}$. A canonical example of such a number is Champernowne's number,

$$
\mathcal{C}_{10}:=0.123456789101112131415161718192021 \cdots,
$$

which given here in base 10, is the size-ordered concatenation of $\mathbb{N}$ (each number written in base 10) proceeded by a decimal point. Champernowne's number was shown to be 10-normal by Champernowne [5] in 1933 and transcendental by Mahler 9] in 1937.

In 1973, Stoneham [12] defined the following class of numbers. Let $b, c \geqslant 2$ be relatively prime integers. The Stoneham number $\alpha_{b, c}$ is given by

$$
\alpha_{b, c}:=\sum_{n \geqslant 1} \frac{1}{c^{n} b^{c^{n}}} .
$$

Stoneham [12] showed that $\alpha_{2,3}$ is 2-normal. A new proof of this result was given by Bailey and Misiurewicz [4] and finally in 2002, Bailey and Crandall [3] proved that $\alpha_{b, c}$ is $b$-normal for all coprime integers $b, c \geqslant 2$; see also Bailey and Borwein 2. Transcendence of $\alpha_{b, c}$ follows easily by Mahler's method; the interested reader can see the details Appendix $\mathrm{A}$.

Recently Francisco Aragón Artacho, David Bailey, Jonathan Borwein, and Peter Borwein [1] made two conjectures concerning properties of the base- 4 expansion of the Stoneham number $\alpha_{2,3}$ and the base-3 expansion of $\alpha_{3,5}$, respectively. In this paper, we prove their conjectures, and as such they are stated here as theorems (we have fixed a few small typos in their published conjectures).

Date: June 26, 2018.

Key words and phrases. Stoneham numbers, base- $b$ expansions, normal numbers.

The research of M. Coons is supported by Australian Research Council grant DE140100223. 
Theorem 1.1. Let the base-4 expansion of $\alpha_{2,3}$ be given by $\alpha_{2,3}:=\sum_{k \geqslant 1} d_{k} 4^{-k}$, with $d_{k} \in\{0,1,2,3\}$. Then for all $n \geqslant 0$ one has

$$
\sum_{k=\frac{3}{2}\left(3^{n}+1\right)}^{\frac{3}{2}\left(3^{n}+1\right)+3^{n}-1}\left(e^{\frac{\pi i}{2}}\right)^{d_{k}}=- \begin{cases}i, & \text { if } n \text { is odd } \\ 1, & \text { if } n \text { is even }\end{cases}
$$

(ii) $d_{k}=d_{3^{n}+k}=d_{2 \cdot 3^{n}+k}$ for $k=\frac{3}{2}\left(3^{n}+1\right), \frac{3}{2}\left(3^{n}+1\right)+1, \ldots, \frac{3}{2}\left(3^{n}+1\right)+3^{n}-1$.

Theorem 1.2. Let the base-3 expansion of $\alpha_{3,5}$ be given by $\alpha_{3,5}:=\sum_{k \geqslant 1} a_{k} 3^{-k}$, with $a_{k} \in\{0,1,2\}$. Then for all $n \geqslant 0$ one has

(i) $\sum_{k=1+5^{n+1}}^{1+5^{n+1}+4 \cdot 5^{n}}\left(e^{\frac{\pi i}{3}}\right)^{a_{k}}=(-1)^{n} e^{\frac{\pi i}{3}}$

(ii) $a_{k}=a_{4 \cdot 5^{n}+k}=a_{8 \cdot 5^{n}+k}=a_{12 \cdot 5^{n}+k}=a_{16 \cdot 5^{n}+k}$ for $k=5^{n+1}+j$, with $j=1, \ldots, 4 \cdot 5^{n}$.

We note here that the Stoneham numbers $\alpha_{b, c}$ are in some ways very similar to Champernowne's numbers. They are not concatenations of consecutive integers, but the concatenation of periods of certain rational numbers. Let $b, c \geqslant 2$ be coprime integers and let $w_{n}$ be the word $w \in\{0,1, \ldots, b-1\}^{*}$ of minimal length such that

$$
\left(\frac{1}{c^{n}}\right)_{b}=0 . \overline{w_{n}}
$$

where $(x)_{b}$ denotes the base- $b$ expansion of the real number $x$ and $\bar{w}$ denotes the infinitely repeated word $w$. Then the Stoneham numbers are similar to the numbers

$$
0 . w_{1} w_{2} w_{3} w_{4} w_{5} \cdots w_{n} \cdots,
$$

which are given by concatenating the words $w_{n}$. Indeed, the Stoneham number has this structure, but with the $w_{j}$ repeated and cyclicly shifted.

Remark 1.3. While we will be considering the base- 4 expansion of $\alpha_{2,3}$ we are still dealing with a normal number; $\alpha_{2,3}$ is also 4-normal. This is given by a result of Schmidt [11] who proved in 1960 that the $r$-normal real number $x$ is $s$-normal if $\log r / \log s \in \mathbb{Q}$.

\section{BASE- $b$ EXPANSIONS OF RATIONALS}

To prove the above theorems in as much generality as possible we will need to consider how we write a reduced fraction $a / k$ in the base $b$. Such an algorithm is well-known, but we remind the reader here, as it will be useful to have the general framework for the proofs of Theorems 1.1 and 1.2. To write $a / k$ in the base $b$, we use a sort of modified division algorithm; see Figure 1 .

We record here facts about the base- $b$ algorithm, which we will need.

Lemma 2.1. Suppose $b, k \geqslant 2$ are coprime, and that $r_{j}$ and $q_{j}$ are defined by the base-b algorithm for $a / k$. Then $\operatorname{gcd}\left(r_{i}, k\right)=1$.

Proof. Suppose that $p \mid k$, and proceed by induction on $i$. Firstly, $r_{0}=a$ and by assumption $\operatorname{gcd}\left(r_{0}, k\right)=\operatorname{gcd}(a, k)=1$.

Now suppose that $\operatorname{gcd}\left(r_{i}, k\right)=1$, so that also $\operatorname{gcd}\left(r_{i} b, k\right)=1$. Then

$$
r_{i+1}=r_{i} b-q_{i+1} k \equiv r_{i} b \not \equiv 0(\bmod p),
$$

since $\operatorname{gcd}(b, k)=1$. Thus $\operatorname{gcd}\left(r_{i+1}, k\right)=1$. 
Base- $b$ Algorithm for $a / k<1$.

Let $b, k \geqslant 2$ be integers and $a \geqslant 1$ be an integer coprime to $k$. Set $r_{0}=a$ and write

$$
\begin{gathered}
r_{0} b=q_{1} k+r_{1} \\
r_{1} b=q_{2} k+r_{2} \\
\vdots \\
r_{j-1} b=q_{j} k+r_{j}
\end{gathered}
$$

where $q_{j} \in\{0,1, \ldots, b-1\}$ and $r_{j} \in\{0,1, \ldots, k-1\}$ for each $j$. Stop when $r_{n}=r_{0}$. Then

$$
\left(\frac{a}{k}\right)_{b}=0 . \overline{q_{1} q_{2} \cdots q_{n}} \text {. }
$$

FiguRE 1 . The base- $b$ algorithm for the reduced rational $a / k<1$.

Also, we have that equivalent $r_{j}$ give equal $q_{j}$.

Lemma 2.2. Suppose $b, k \geqslant 2$ are coprime, and that $r_{j}$ and $q_{j}$ are defined by the base-b algorithm for the reduced fraction $a / k$. We have $r_{i} \equiv r_{j}(\bmod b)$ if and only if $q_{i}=q_{j}$.

Proof. Suppose that $r_{i} \equiv r_{j}(\bmod b)$. By considering the difference between $r_{i-1} b=q_{i} k+r_{i}$ and $r_{j-1} b=q_{j} k+r_{j}$ modulo $b$, we see that $b \mid\left(q_{i}-q_{j}\right) k$, so that since $\operatorname{gcd}(b, k)=1$, we have that $b \mid\left(q_{i}-q_{j}\right)$. Since $q_{i}, q_{j} \in\{0,1, \ldots, b-1\}$, we thus have that $q_{i}=q_{j}$.

Conversely, suppose that $q_{i}=q_{j}$. Here, again, we can consider the difference between the defining equations for $q_{i}$ and $q_{j}$ modulo $b$; this gives the desired result.

Indeed, the value of $q_{j}$ is determined by the residue class of $r_{j}$ modulo $b$ and the value of $k^{-1}$ modulo $b$.

Lemma 2.3. Suppose $b, k \geqslant 2$ are coprime, and that $r_{j}$ and $q_{j}$ are defined by the base $b$ algorithm for the reduced fraction $a / k$. We have $r_{i} \equiv j(\bmod b)$ if and only if $q_{i} \equiv-j k^{-1}(\bmod b)$, where $q_{i} \in\{0,1, \ldots, b-1\}$.

Proof. If $r_{i} \equiv j(\bmod b)$, then the equation $r_{i-1} b=q_{i} k+r_{i}$ gives $q_{i} k \equiv-j(\bmod$ $b)$, which in turn gives that $q_{i} \equiv-j k^{-1}(\bmod b)$. Since $q_{i} \in[0, b-1]$ we are done with this direction of proof.

Conversely, suppose that $q_{i}=\left(-j k^{-1} \bmod b\right)$. Then surely, $q_{i} \equiv-j k^{-1}(\bmod b)$ and so $q_{i} k \equiv-j(\bmod b)$. Thus, again using $r_{i-1} b=q_{i} k+r_{i}$, we have that $r_{i} \equiv$ $j(\bmod b)$.

The following Lemma is a direct corollary of Lemma 2.3 .

Lemma 2.4. Suppose $b, k \geqslant 2$ are coprime, and that $r_{j}$ and $q_{j}$ are defined by the base $b$ algorithm for the reduced fraction $a / k$. We have $r_{i} \equiv 0(\bmod b)$ if and only if $q_{i}=0$. 
Proof. Apply Lemma 2.3 with $j=0$.

We will use the following classical theorem (see [10, Theorem 12.4]) and lemma.

Theorem 2.5. Let $b$ be a positive integer. Then the base $b$ expansion of a rational number either terminates or is periodic. Further, if $r, s \in \mathbb{Z}$ with $0<r / s<1$ where $\operatorname{gcd}(r, s)=1$ and $s=T U$, where every prime factor of $T$ divides $b$ and $\operatorname{gcd}(U, b)=1$, then the period length of the base-b expansion of $r / s$ is the order of $b$ modulo $U$, and the preperiod length is $N$ where $N$ is the smallest positive integer such that $T \mid b^{N}$.

Theorem 2.5 tells us that the base $b$ expansion of $a / k$ is purely periodic (recall for us $\operatorname{gcd}(b, k)=1$ ), and that the minimal period is $\operatorname{ord}_{k} b$, which divides $\varphi(k)$, so that this also is a period. This result can be exploited using the following numbertheoretic result, a proof of which can be found in most elementary number theory texts, e.g., see [10, Theorem 9.10].

Lemma 2.6. A primitive root of $p^{2}$ is a primitive root of $p^{k}$ for any integer $k \geqslant 2$.

Applying Lemma 2.6 gives the following result.

Lemma 2.7. Let $0<a / p^{m}<1$ be a rational number in lowest terms and let $b \geqslant 2$ be an integer that is a primitive root of $p^{2}$. Suppose that $\left(1 / p^{m}\right)_{b}=. \overline{q_{1} q_{2} \cdots q_{n}}$ is given by the base $b$ algorithm. Then

$$
\left(\frac{a}{p^{m}}\right)_{b}=. \overline{q_{\sigma(1)} q_{\sigma(2)} \cdots q_{\sigma(n)}}
$$

where $\sigma$ is a cyclic shift on $n$ letters.

Proof. This is a direct consequence of the base- $b$ algorithm.

As a consequence of the above lemmas we are able to provide the following characterisation of certain base- $b$ expansions.

Proposition 2.8. Let $m \geqslant 1$ be an integer, $p$ be an odd prime, $b \geqslant 2$ be an integer coprime to $p$, and $q_{j}$ and $r_{j}$ be given by the base-b algorithm for the reduced fraction $a / p^{m}$. If $b$ is a primitive root of $p$ and $p^{2}$, then $\operatorname{period}\left(a / p^{m}\right)=\varphi\left(p^{m}\right)$ and

$$
\#\left\{j \leqslant \varphi\left(p^{m}\right): q_{j}=0\right\}=\left\lfloor\frac{p^{m}}{b}\right\rfloor-\left\lfloor\frac{p^{m-1}}{b}\right\rfloor .
$$

Proof. The fact that period $\left(a / p^{m}\right)_{b}=\varphi\left(p^{m}\right)$ follows directly from $b$ being a primitive root of $p$ and $p^{2}$, Lemma 2.6 and Theorem 2.5. This further implies that the $\varphi\left(p^{m}\right)$ values of $r_{i}$ given by the base- $b$ algorithm for $a / p^{m}$ are distinct. Applying Lemma 2.1 gives that

$$
\left\{r_{1}, r_{2}, \ldots, r_{\varphi\left(p^{m}\right)}\right\}=\left\{i \leqslant p^{m}: \operatorname{gcd}(i, p)=1\right\} .
$$

Also recall that

$$
\left(\frac{a}{p^{m}}\right)_{b}=\overline{q_{1} q_{2} \cdots q_{\varphi\left(p^{m}\right)}},
$$

and that by Lemma 2.4, $q_{i}=0$ if and only if $r_{i} \equiv 0(\bmod b)$. Note that there are exactly

$$
\left\lfloor\frac{p^{m}}{b}\right\rfloor-\left\lfloor\frac{p^{m}}{b p}\right\rfloor=\left\lfloor\frac{p^{m}}{b}\right\rfloor-\left\lfloor\frac{p^{m-1}}{b}\right\rfloor
$$


elements of $\left\{i \leqslant p^{m}: \operatorname{gcd}(i, p)=1\right\}$ which are divisible by $b$. Thus using the set equality (11), we have that there are exactly $\left\lfloor p^{m} / b\right\rfloor-\left\lfloor p^{m-1} / b\right\rfloor$ elements of $\left\{r_{1}, r_{2}, \ldots, r_{\varphi\left(p^{m}\right)}\right\}$ divisible by $b$. Appealing to Lemma 2.4 we then have that there are $\left\lfloor p^{m} / b\right\rfloor-\left\lfloor p^{m-1} / b\right\rfloor$ of $q_{1}, q_{2}, \ldots, q_{\varphi\left(p^{m}\right)}$ such that $q_{j}=0$.

Note that while we record the $q_{i}=0$ case because of its simplicity, the method can be applied to count any value of $q_{i}$ that is desired by using the appropriate case of Lemma 2.3. In fact, we will do this in a few special cases to prove Theorems 1.1 and 1.2 ,

\section{The Base- $b$ expansion of the Stoneham number $\alpha_{b, p}$}

We will need properties for both the base- $b$ expansion and the base- $b^{2}$ expansions of the Stoneham number $\alpha_{b, p}$.

Proposition 3.1. Let $b, p \geqslant 2$ be coprime integers with $p$ a prime. Denote the base-b expansion of $\alpha_{b, p}$ as

$$
\alpha_{b, p}=\sum_{j \geqslant 1} \frac{1}{p^{j} b^{p^{j}}}=\sum_{k \geqslant 1} \frac{a_{k}}{b^{k}}
$$

where $a_{k} \in\{0,1, \ldots, b-1\}$ and write

$$
\left(\frac{\sum_{j=0}^{m-1} p^{j}}{p^{m}}\right)_{b}=\overline{q_{1} q_{2} \cdots q_{n}}
$$

where $q_{i}$ is determined by the base-b algorithm, for each $i$, so $n=\operatorname{ord}_{p^{m}} b$. Then $q_{i}=a_{p^{m}+j n+i}$ for each $i \in\{1,2, \ldots, n\}$ and each $j \in\left\{0,1,2, \ldots, \frac{p \cdot \varphi\left(p^{m}\right)}{\operatorname{ord}_{p} m b}-1\right\}$.

It is worth noting that Propositions 3.1] is the full generalisation of Theorem[1.1(ii).

We require the following lemma.

Lemma 3.2. Let $b, c \geqslant 2$ be coprime. Then for any $m \geqslant 1$ we have

$$
\alpha_{b, c}-\sum_{n=1}^{m} \frac{1}{c^{n} b^{c^{n}}}<\frac{1}{b^{c^{m+1}}} .
$$

That is, the base-b expansion of $\alpha_{b, c}$ agrees with the b-ary expansion of its $m$-th partial sum up to the $\left(c^{m+1}\right)$-th place.

Proof. Let $m \geqslant 1$ and note that

$$
\sum_{n \geqslant m+1} \frac{1}{c^{n}}=\frac{1}{c^{m+1}-c^{m}}<1 .
$$

Using this fact, we have that

$$
\alpha_{b, c}-\sum_{n=1}^{m} \frac{1}{c^{n} b^{c^{n}}}=\sum_{n \geqslant m+1} \frac{1}{c^{n} b^{c^{n}}}<\frac{1}{b^{c^{m+1}}} \sum_{n \geqslant m+1} \frac{1}{c^{n}}<\frac{1}{b^{c^{m+1}}},
$$

which is the desired result.

Proof of Proposition 3.1. Let $m \geqslant 1, s_{m}=p^{m} b^{p^{m}}$, and define the positive integer $r_{m}$ by

$$
\frac{r_{m}}{s_{m}}=\sum_{n=1}^{m} \frac{1}{p^{n} b^{p^{n}}} .
$$


We have then that

$$
\operatorname{gcd}\left(r_{m}, s_{m}\right)=\operatorname{gcd}\left(r_{m}, p^{m} b^{p^{m}}\right)=\operatorname{gcd}\left(r_{m}, p b\right)=1 .
$$

We apply Theorem 2.5 with $b=b, r=r_{m}, s=s_{m}, T=b^{p^{m}}$, and $U=p^{m}$ to give that the period length of the base $b$ expansion of $r_{m} / s_{m}$ is the order of $b$ modulo $p^{m}$, which we will write

$$
\operatorname{period}\left(r_{m} / s_{m}\right)=\operatorname{ord}_{p^{m}} b,
$$

and the preperiod length of $r_{m} / s_{m}$ is $p^{m}$, which we will write

$$
\operatorname{preperiod}\left(r_{m} / s_{m}\right)=p^{m} \text {. }
$$

Combining the observations of the previous paragraph with Lemma 3.2 gives that

$$
a_{p^{m}+1} a_{p^{m}+2} \ldots a_{p^{m+1}}=\underbrace{w w w \cdots w}_{\frac{p \cdot \varphi\left(p^{m}\right)}{\operatorname{ord}_{p^{m b}}} \text { times }}
$$

where $w=q_{1} q_{2} \cdots q_{\operatorname{ord}_{p} m b}$ is a word on the alphabet $\{0,1, \ldots, b\}$ with length $\operatorname{ord}_{p^{m}} b$. To finish the proof of this proposition, it is enough to appeal to Lemma 3.2 to show that

$$
\left(\frac{\sum_{j=0}^{m-1} p^{j}}{p^{m}}\right)_{b}=. \bar{w},
$$

where $w$ is as defined in the previous sentence, which follows directly from the definition of $\alpha_{b, p}$.

Theorem 1.1 concerns a base- $b^{2}$ expansion; we will provide some specialised results for this case, only when $b=2$, in order to specifically prove Theorem 1.1. as the more interesting case for generalisations is the base- $b$ case.

Lemma 3.3. Let $b, c \geqslant 2$ be coprime. Then for any $m \geqslant 1$ we have

$$
\alpha_{b, c}-\sum_{n=1}^{m} \frac{1}{c^{n} b^{c^{n}}}<\frac{1}{\left(b^{2}\right)^{c^{m+1} / 2}} .
$$

That is, the base- $b^{2}$ expansion of $\alpha_{b, c}$ agrees with the base- $b^{2}$ expansion of its $m$-th partial sum up to the $\left\lceil c^{m+1} / 2\right\rceil$-th place.

Proof. This is a direct consequence of Lemma 3.2 .

Proposition 3.4. Let $p$ be an odd prime such that 2 is a primitive root of $p$ and $p^{2}$. Denote the base-4 expansion of $\alpha_{2, p}$ as

$$
\alpha_{2, p}=\sum_{j \geqslant 1} \frac{1}{p^{j} 2^{p^{j}}}=\sum_{k \geqslant 1} \frac{d_{k}}{4^{k}},
$$

where $d_{k} \in\{0,1, \ldots, 3\}$ and write

$$
\left(\frac{\sum_{j=0}^{m-1} p^{j}}{p^{m}}\right)_{4}=\overline{q_{1} q_{2} \cdots q_{n}}
$$

where the $q_{i}$ s are determined by the base-4 algorithm, so $n=\operatorname{ord}_{p^{m}} 4=\varphi\left(p^{m}\right) / 2$. Then $q_{i}=d_{\frac{p^{m}+1}{2}+j n+i}$ for each $i \in\{1, \ldots, n\}$ and each $j \in\{0,1,2, \ldots, p-1\}$. 
Proof. This proposition follows as a corollary of Proposition 3.1 Indeed, by Proposition 3.1, we have a prefix $u$ of odd length $p$ and words $w_{m}$ of even length $\varphi\left(p^{m}\right)$ such that

$$
\left(\alpha_{2, p}\right)_{2}=. u \underbrace{w_{1} w_{1} \cdots w_{1}}_{p \text { times }} \underbrace{w_{2} w_{2} \cdots w_{2}}_{p \text { times }} \cdots \underbrace{w_{m} w_{m} \cdots w_{m}}_{p \text { times }} \cdots .
$$

Now the word $w_{m}$ is the minimal repeated word given by the base- 2 expansion of $\left(\sum_{j=0}^{m-1} p^{j}\right) / p^{m}$. But

$$
0<\frac{\sum_{j=0}^{m-1} p^{j}}{p^{m}}=\frac{p^{m}-1}{p^{m}(p-1)}<\frac{1}{p-1} \leqslant \frac{1}{2}
$$

and so the first letter of $w_{m}$, for each $m$, is necessarily 0 . Define the word $v_{m}$ by $w_{m}=0 v_{m}$. Then

$$
\begin{aligned}
& \left(\alpha_{2, p}\right)_{2}=. u \underbrace{w_{1} w_{1} \cdots w_{1}}_{p \text { times }} \underbrace{w_{2} w_{2} \cdots w_{2}}_{p \text { times }} \cdots \underbrace{w_{m} w_{m} \cdots w_{m}}_{p \text { times }} \cdots \\
& =. u \underbrace{0 v_{1} 0 v_{1} \cdots 0 v_{1}}_{p \text { times }} \underbrace{0 v_{2} 0 v_{2} \cdots 0 v_{2}}_{p \text { times }} \cdots \underbrace{0 v_{m} 0 v_{m} \cdots 0 v_{m}}_{p \text { times }} \cdots \\
& =. u 0 \underbrace{v_{1} 0 v_{1} 0 \cdots v_{1} 0}_{p \text { times }} \underbrace{v_{2} 0 v_{2} 0 \cdots v_{2} 0}_{p \text { times }} \cdots \underbrace{v_{m} 0 v_{m} 0 \cdots v_{m} 0}_{p \text { times }} \cdots,
\end{aligned}
$$

where we have that the word $u 0$ is of even length $p+1$ and the word $v_{m} 0$ is of even length $\varphi\left(p^{m}\right)$.

As in the statement of Proposition 3.1, let $a_{k}$ be the $k$ th letter in the base-2 expansion of $\alpha_{2, p}$, and as in the statement of the current proposition, let $d_{k}$ be the $k$ th letter in the base- 4 expansion of $\alpha_{2, p}$. Then

$$
d_{k}=2 a_{2 k-1}+a_{2 k} .
$$

Using this fact, it is an immediate consequence of (3) that there are words $U$ of length $(p+1) / 2$ and $W_{m}$ of length $\varphi\left(p^{m}\right) / 2$ such that

$$
\left(\alpha_{2, p}\right)_{4}=. U \underbrace{W_{1} W_{1} \cdots W_{1}}_{p \text { times }} \underbrace{W_{2} W_{2} \cdots W_{2}}_{p \text { times }} \cdots \underbrace{W_{m} W_{m} \cdots W_{m}}_{p \text { times }} \cdots .
$$

As in Proposition 3.1, to finish the proof of this proposition, it is enough to apply Lemma 3.3 to show that

$$
\left(\frac{\sum_{j=0}^{m-1} p^{j}}{p^{m}}\right)_{4}=. \overline{W_{m}},
$$

where $W_{m}$ is as defined in the previous sentence, which follows directly from the definition of $\alpha_{2, p}$.

\section{The Aragon, Borwein, Borwein, and Bailey Conjectures}

In this section, we apply the results of Section 3 to prove Theorems 1.1 and 1.2 As is turns out, the proof of Theorem 1.2 is a bit more straightforward, so we present its proof first. 
Proof of Theorem 1.2. For convenience let us write $\omega:=e^{\pi i / 3}$ and let $r_{i}$ and $q_{i}$ be given by the base- 3 algorithm for $1 / 5^{n}$. Note that by Proposition 3.1, we have that

$$
\sum_{k=1+5^{n+1}}^{1+5^{n+1}+4 \cdot 5^{n}} \omega^{a_{k}}=\sum_{j=0}^{2} \#\left\{i \leqslant \varphi\left(5^{n+1}\right): q_{i}=j\right\} \cdot \omega^{j} .
$$

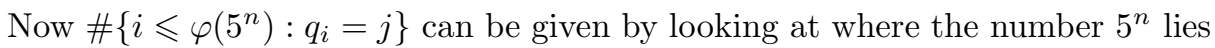
modulo 15 . Since, for every 15 consecutive numbers, 12 of them are coprime to 5 , and these 12 fall into the 3 equivalence classes modulo 3 with an equal frequency of 4 times each, we need only look at the remainder of $5^{n}$ modulo 15 . An easy calculation gives that

$$
5^{n} \equiv \begin{cases}5(\bmod 15) & \text { if } n \text { is odd } \\ 10(\bmod 15) & \text { if } n \text { is even }\end{cases}
$$

This allows us to give that

$$
\begin{aligned}
& \#\left\{i \leqslant \varphi\left(5^{n}\right): r_{i} \equiv 0(\bmod 3)\right\}= \begin{cases}4 \cdot\left\lfloor\frac{5^{n}}{15}\right\rfloor+1 & \text { if } n \text { is odd } \\
4 \cdot\left\lfloor\frac{5^{n}}{15}\right\rfloor+3 & \text { if } n \text { is even, }\end{cases} \\
& \#\left\{i \leqslant \varphi\left(5^{n}\right): r_{i} \equiv 1(\bmod 3)\right\}= \begin{cases}4 \cdot\left\lfloor\frac{5^{n}}{15}\right\rfloor+2 & \text { if } n \text { is odd } \\
4 \cdot\left\lfloor\frac{5^{n}}{15}\right\rfloor+3 & \text { if } n \text { is even, }\end{cases}
\end{aligned}
$$

and

$$
\#\left\{i \leqslant \varphi\left(5^{n}\right): r_{i} \equiv 2(\bmod 3)\right\}= \begin{cases}4 \cdot\left\lfloor\frac{5^{n}}{15}\right\rfloor+1 & \text { if } n \text { is odd } \\ 4 \cdot\left\lfloor\frac{5}{15}\right\rfloor+2 & \text { if } n \text { is even. }\end{cases}
$$

Applying Lemma 2.3 to the preceding equalities gives that

$$
\begin{aligned}
& \#\left\{i \leqslant \varphi\left(5^{n}\right): q_{i}=0\right\}= \begin{cases}4 \cdot\left\lfloor\frac{5^{n}}{15}\right\rfloor+1 & \text { if } n \text { is odd } \\
4 \cdot\left\lfloor\frac{5^{n}}{15}\right\rfloor+3 & \text { if } n \text { is even, }\end{cases} \\
& \#\left\{i \leqslant \varphi\left(5^{n}\right): q_{i}=1\right\}= \begin{cases}4 \cdot\left\lfloor\frac{5^{n}}{15}\right\rfloor+2 & \text { if } n \text { is odd } \\
4 \cdot\left\lfloor\frac{5^{n}}{15}\right\rfloor+2 & \text { if } n \text { is even, }\end{cases}
\end{aligned}
$$

and

$$
\#\left\{i \leqslant \varphi\left(5^{n}\right): q_{i}=2\right\}= \begin{cases}4 \cdot\left\lfloor\frac{5^{n}}{15}\right\rfloor+1 & \text { if } n \text { is odd } \\ 4 \cdot\left\lfloor\frac{5^{n}}{15}\right\rfloor+3 & \text { if } n \text { is even. }\end{cases}
$$

Since $1+\omega+\omega^{2}=0$, we thus have that

$$
\begin{aligned}
& \sum_{k=1+5^{n+1}}^{1+5^{n+1}+4 \cdot 5^{n}} \omega^{a_{k}}=\sum_{j=0}^{2} \#\left\{i \leqslant \varphi\left(5^{n+1}\right): q_{i}=j\right\} \cdot \omega^{j} \\
& = \begin{cases}\omega & \text { if } n+1 \text { is odd } \\
-\omega & \text { if } n+1 \text { is even }\end{cases} \\
& =(-1)^{n} \omega \text {, }
\end{aligned}
$$

which proves part (i).

Part (ii) follows directly from Proposition 3.1 with $b=3$ and $p=5$. 
Proof of Theorem 1.1. Note that

$$
\frac{1}{3^{n} 2^{3^{n}}}=\frac{8}{3^{n}} \cdot \frac{1}{4^{\frac{3}{2}\left(3^{n-1}+1\right)}} .
$$

Let $r_{i}$ and $q_{i}$ be given by the base 4 algorithm for $8 / 3^{n}$. We will use the fact that each of these $r_{i}$ is equivalent to 2 modulo 3 . This is easily seen as we have for each $i$ that $r_{i-1} 4=q_{i} 3^{n}+r_{i}$, so that taking this equality modulo 3 we have that $r_{i-1} \equiv r_{i}(\bmod 3)$. Recalling that $r_{0}=8$ shows that indeed $r_{i} \equiv 2(\bmod 3)$ for each $i$.

Since $\operatorname{ord}_{3^{n}} 4=3^{n-1}$, we have, by Proposition 3.4, that

$$
\sum_{k=\frac{3}{2}\left(3^{n}+1\right)}^{\frac{3}{2}\left(3^{n}+1\right)+3^{n}-1}\left(e^{\frac{\pi i}{2}}\right)^{a_{k}}=\sum_{j=0}^{3} \#\left\{i \leqslant \varphi\left(3^{n+1}\right) / 2: q_{i}=j\right\} \cdot\left(e^{\frac{\pi i}{2}}\right)^{j} .
$$

Now $\#\left\{i \leqslant 3^{n}: q_{i}=j\right\}$ can be given by looking at where the number $3^{n}$ lies modulo 12 . Since, for every 12 consecutive numbers, 4 of them are equivalent to 2 modulo 3 , and these 4 fall into the 4 distinct equivalence classes modulo 4 , we must consider the remainder of $3^{n}$ modulo 12 . We have that

$$
3^{n} \equiv \begin{cases}3(\bmod 12) & \text { if } n \text { is odd } \\ 9(\bmod 12) & \text { if } n \text { is even. }\end{cases}
$$

Thus we have that

$$
\begin{aligned}
& \#\left\{i \leqslant \varphi\left(3^{n}\right) / 2: r_{i} \equiv 0(\bmod 4)\right\}= \begin{cases}\left\lfloor\frac{3^{n}}{12}\right\rfloor & \text { if } n \text { is odd } \\
\left\lfloor\frac{3^{n}}{12}\right\rfloor+1 & \text { if } n \text { is even, }\end{cases} \\
& \#\left\{i \leqslant \varphi\left(3^{n}\right) / 2: r_{i} \equiv 1(\bmod 4)\right\}= \begin{cases}\left\lfloor\frac{3^{n}}{12}\right\rfloor & \text { if } n \text { is odd } \\
\left\lfloor\frac{3^{n}}{12}\right\rfloor+1 & \text { if } n \text { is even, }\end{cases} \\
& \#\left\{i \leqslant \varphi\left(3^{n}\right) / 2: r_{i} \equiv 2(\bmod 4)\right\}= \begin{cases}\left\lfloor\frac{3^{n}}{12}\right\rfloor+1 & \text { if } n \text { is odd } \\
\left\lfloor\frac{3^{n}}{12}\right\rfloor+1 & \text { if } n \text { is even, }\end{cases}
\end{aligned}
$$

and

$$
\#\left\{i \leqslant \varphi\left(3^{n}\right) / 2: r_{i} \equiv 3(\bmod 4)\right\}= \begin{cases}\left\lfloor\frac{3^{n}}{12}\right\rfloor & \text { if } n \text { is odd } \\ \left\lfloor\frac{3^{n}}{12}\right\rfloor & \text { if } n \text { is even. }\end{cases}
$$

By Lemma 2.3, we have that

$$
\begin{aligned}
& \#\left\{i \leqslant \varphi\left(3^{n}\right) / 2: q_{i}=0\right\}= \begin{cases}\left\lfloor\frac{3^{n}}{12}\right\rfloor & \text { if } n \text { is odd } \\
\left\lfloor\frac{3^{n}}{12}\right\rfloor+1 & \text { if } n \text { is even, }\end{cases} \\
& \#\left\{i \leqslant \varphi\left(3^{n}\right) / 2: q_{i}=1\right\}= \begin{cases}\left\lfloor\frac{3^{n}}{12}\right\rfloor \text { if } n \text { is odd } \\
\left\lfloor\frac{3^{n}}{12}\right\rfloor \text { if } n \text { is even, }\end{cases} \\
& \#\left\{i \leqslant \varphi\left(3^{n}\right) / 2: q_{i}=2\right\}= \begin{cases}\left\lfloor\left\lfloor\frac{3^{n}}{12}\right\rfloor+1\right. & \text { if } n \text { is odd } \\
\left\lfloor\frac{3^{n}}{12}\right\rfloor+1 & \text { if } n \text { is even, }\end{cases}
\end{aligned}
$$

and

$$
\#\left\{i \leqslant \varphi\left(3^{n}\right) / 2: q_{i}=3\right\}= \begin{cases}\left\lfloor\frac{3^{n}}{12}\right\rfloor & \text { if } n \text { is odd } \\ \left\lfloor\frac{3^{n}}{12}\right\rfloor+1 & \text { if } n \text { is even. }\end{cases}
$$


Since $1+\left(e^{\frac{\pi i}{2}}\right)+\left(e^{\frac{\pi i}{2}}\right)^{2}+\left(e^{\frac{\pi i}{2}}\right)^{3}=0$, we thus have that

$$
\begin{aligned}
\sum_{k=\frac{3}{2}\left(3^{n}+1\right)}^{\frac{3}{2}\left(3^{n}+1\right)+3^{n}-1}\left(e^{\frac{\pi i}{2}}\right)^{a_{k}} & =\sum_{j=0}^{3} \#\left\{i \leqslant \varphi\left(3^{n+1}\right) / 2: q_{i}=j\right\} \cdot\left(e^{\frac{\pi i}{2}}\right)^{j} \\
& = \begin{cases}-1 & \text { if } n+1 \text { is odd } \\
-i & \text { if } n+1 \text { is even }\end{cases} \\
& =- \begin{cases}i & \text { if } n \text { is odd } \\
1 & \text { if } n \text { is even },\end{cases}
\end{aligned}
$$

which proves part (i).

Part (ii) follows directly from Proposition 3.4 with $b=2$ and $p=3$.

Acknowledgements. To be written ...

\section{Appendix A. Transcendence of Stoneham numbers}

In this appendix, we give details of the transcendence of the Stoneham number $\alpha_{b, c}$ for any choice of integers $b, c \geqslant 2$. In fact, Mahler's method gives much stronger results, which imply this desired conclusion.

We start out by letting $c \geqslant 2$ be an integer and define

$$
F_{c}(x):=\sum_{n \geqslant 1} \frac{x^{c^{n}}}{c^{n}} .
$$

Notice that $F_{c}(x)$ satisfies the Mahler functional equation

$$
F_{c}\left(x^{c}\right)=c F_{c}(x)-x^{c} .
$$

Now suppose that $F_{c}(x) \in \mathbb{C}(x)$. Then there are polynomials $a(x), b(x) \in \mathbb{C}[x]$ such that

$$
F_{c}(x)-\frac{a(x)}{b(x)}=0
$$

Since $F_{c}(x) \in \mathbb{C}[[x]]$ is not a polynomial, we may assume, without loss of generality, that $\operatorname{gcd}(a(x), b(x))=1$ and $b(0) \neq 0$ and $b(x) \notin \mathbb{C}$. Sending $x \rightarrow x^{c}$ and applying the functional equation, we thus have that

$$
F_{c}(x)-\frac{a(x)}{b(x)}=0=F_{c}\left(x^{c}\right)-\frac{a\left(x^{c}\right)}{b\left(x^{c}\right)}=F_{c}(x)-\left(\frac{x^{c}}{c}+\frac{a\left(x^{c}\right)}{b\left(x^{c}\right)}\right),
$$

so that

$$
\frac{x^{c}}{c}+\frac{a\left(x^{c}\right)}{b\left(x^{c}\right)}=\frac{a(x)}{b(x)} .
$$

Now as functions, the righthand and lefthand sides of the equation in (5) must have the same singularities. But $b\left(x^{c}\right)$ will have more zeros (counting multiplicity if needed) than $b(x)$ unless $b(x)$ is a constant, which is a contradiction. Thus $F_{c}(x)$ does not represent a rational function. In fact, we can now appeal to the following theorem, to give that $F_{c}(x)$ is transcendental over $\mathbb{C}(x)$. 
Theorem A.1 (Nishioka, 1985). Suppose that $F(x) \in \mathbb{C}[[x]]$ satisfies one of the following for an integer $d>1$.

(i) $F\left(x^{d}\right)=\phi(x, F(x))$,

(ii) $F(x)=\phi\left(x, F\left(x^{d}\right)\right)$,

where $\phi(x, u)$ is a rational function in $x, u$ over $\mathbb{C}$. If $F(x)$ is algebraic over $\mathbb{C}(x)$, then $F(x) \in \mathbb{C}(x)$.

To prove the transcendence of the Stoneham numbers, we appeal to a classical result of Mahler [8], We record it here as taken from Nishioka's mongraph Mahler Functions and Transcendence [7.

Theorem A.2 (Mahler [8]). Let $\mathbf{I}$ be the set of algebraic integers over $\mathbb{Q}, K$ be an algebraic number field, $\mathbf{I}_{K}=K \cap \mathbf{I}, f(x) \in K[[x]]$ with radius of convergence $R>0$ satisfying the functional equation for an integer $d>1$,

$$
f\left(x^{d}\right)=\frac{\sum_{i=0}^{m} a_{i}(x) f(x)^{i}}{\sum_{i=0}^{m} b_{i}(x) f(x)^{i}}, \quad m<d, a_{i}(x), b_{i}(x) \in \mathbf{I}_{K}[x]
$$

and $\Delta(x):=\operatorname{Res}(A, B)$ be the resultant of $A(u)=\sum_{i=0}^{m} a_{i}(x) u^{i}$ and $B(u)=$ $\sum_{i=0}^{m} b_{i}(x) u^{i}$ as polynomials in $u$. If $f(x)$ is transcendental over $K(x)$ and $\xi$ is an algebraic number with $0<|\xi|<\min \{1, R\}$ and $\Delta\left(\xi^{d^{n}}\right) \neq 0(n \geqslant 0)$, then $f(\xi)$ is transcendental.

Since $F_{c}(x)$ is transcendental over $\mathbb{C}(x), F_{c}(x)$ satisfies the functional equation (44), and $\operatorname{Res}\left(c u-x^{c}, 1\right) \neq 0$ for all $x$, we have the following corollary to Mahler's theorem.

Corollary A.3. Let $c \geqslant 2$ be an integer. The number $\sum_{n \geqslant 1} \frac{1}{c^{n}} \xi^{c^{n}}$ is transcendental for all algebraic numbers $\xi$ with $0<|\xi|<1$. In particular, for all $b, c \geqslant 2$, the number Stoneham number $\alpha_{b, c}$ is transcendental.

\section{REFERENCES}

[1] Francisco J. Aragón Artacho, David H. Bailey, Jonathan M. Borwein, and Peter B. Borwein, Walking on real numbers, Math. Intelligencer, to appear.

[2] David H. Bailey and Jonathan M. Borwein, Normal numbers and pseudorandom generators, Proceedings of the Workshop on Computational and Analytical Mathematics in Honour of Jonathan Borwein's 60th Birthday, Springer, Berlin, 2012, p. in press.

[3] David H. Bailey and Richard E. Crandall, Random generators and normal numbers, Experiment. Math. 11 (2002), no. 4, 527-546 (2003).

[4] David H. Bailey and Michał Misiurewicz, A strong hot spot theorem, Proc. Amer. Math. Soc. 134 (2006), no. 9, 2495-2501.

[5] D. G. Champernowne, The Construction of Decimals Normal in the Scale of Ten, J. London Math. Soc. 8 (1933), no. 4, 254.

[6] Keiji Nishioka, Algebraic function solutions of a certain class of functional equations, Arch. Math. 44 (1985), 330-335.

[7] Kumiko Nishioka, Mahler functions and transcendence, Lecture Notes in Mathematics, vol. 1631, Springer-Verlag, Berlin, 1996.

[8] Kurt Mahler, Arithmetische Eigenschaften der Lösungen einer Klasse von Funktionalgleichungen, Math. Ann. 101 (1929), no. 1, 342-366.

[9] Kurt Mahler, Arithmetische Eigenschaften einer Klasse von Dezimalbrüchen, Proc. Kon. Nederlandsche Akad. v. Wetenschappen 40 (1937), 421-428.

[10] Kenneth H. Rosen, Elementary number theory and its applications, fifth ed., Addison-Wesley, Reading, MA, 2005.

[11] Wolfgang M. Schmidt, On normal numbers, Pacific J. Math. 10 (1960), 661-672. 
[12] R. G. Stoneham, On absolute $(j, \varepsilon)$-normality in the rational fractions with applications to normal numbers, Acta Arith. 22 (1972/73), 277-286.

School of Mathematical and Physical Sciences, University of Newcastle, University Drive, Callaghan NSW 2300, Australia

E-mail address: Michael.Coons@newcastle.edu.au 